\title{
A UNIFIED EXISTENCE AND UNIQUENESS THEOREM FOR STOCHASTIC EVOLUTION EQUATIONS
}

\author{
A. JENTZEN and P. E. KLOEDEN ${ }^{凶}$
}

(Received 11 December 2008)

\begin{abstract}
An existence and uniqueness theorem for mild solutions of stochastic evolution equations is presented and proved. The diffusion coefficient is handled in a unified way which allows a unified theorem to be formulated for different cases, in particular, of multiplicative space-time white noise and trace-class noise.
\end{abstract}

2000 Mathematics subject classification: primary 60H15; secondary 35K90.

Keywords and phrases: stochastic partial differential equation, existence and uniqueness theorem, spacetime white noise, trace-class noise.

\section{Introduction}

The theory of stochastic evolution equations, by which we mean essentially stochastic partial differential equations (SPDEs) of the parabolic or hyperbolic types, shares all of the complications of the corresponding theory for their deterministic counterparts together with many more due to the nature of the noise, in particular its infinite dimensionality. The literature contains many existence and uniqueness results for such SPDEs; see, for example, Da Prato and Zabczyk [3, 4] and Prévot and Röckner [9] and the papers cited therein.

In this note we consider SPDEs of the form

$$
d X_{t}=\left[A X_{t}+F\left(X_{t}\right)\right] d t+B\left(X_{t}\right) d W_{t}, \quad X_{0}=x_{0}
$$

on a Hilbert space $H$ and where $A$ is in general an unbounded linear operator (for example $A=\Delta$ with Dirichlet boundary conditions), $F$ and $B$ are (possibly) nonlinear functions and $W_{t}$ is a cylindrical Wiener process. In particular, we consider the

This work has been supported by the DFG project 'Pathwise numerics and dynamics of stochastic evolution equations'.

(C) 2009 Australian Mathematical Publishing Association Inc. 0004-9727/2009 \$16.00 
SPDE (1.1) in the mild integral equation form

$$
X_{t}=e^{A t} x_{0}+\int_{0}^{t} e^{A(t-s)} F\left(X_{s}\right) d s+\int_{0}^{t} e^{A(t-s)} B\left(X_{s}\right) d W_{s}, \quad \text { almost surely, }
$$

for $t \in[0, T]$. A precise description of that equation and the assumptions that we use are given in Section 2.

In this article we concentrate in particular on the diffusion coefficient. We assume that $B: H \rightarrow L(U, D)$ is a strongly measurable mapping from $H$ into the space of bounded linear operators from $U$ to $D$, where $D$ is a (possibly) larger space than $H$. In addition we assume $e^{A t}(D) \subset H$ for every $t>0$ and therefore $e^{A t} B(v)$ is a welldefined linear operator from $U$ to $H$ for every $v \in H, t>0$. Then, instead of Lipschitz assumptions on $B$ directly, we assume that the mapping $v \mapsto e^{A t} B(v)$ satisfies a linear growth bound and a global Lipschitz condition with respect to the Hilbert-Schmidt norm for each $t>0$ with the constants depending of a fractional power of $t$ (see also [4, Theorem 5.3.1]). This enables us to handle space-time white noise and trace-class noise as well as additive and multiplicative noise in one setting. This is useful when, for example, one investigates Taylor expansions and numerical approximations of the solutions of such SPDEs [5, 6, 8].

This article is organized as follows. In the next section, we describe more exactly the SPDE that we are considering and state the assumptions that we require on its terms and coefficients and on the initial value. We state our existence and uniqueness theorem and a second theorem on the regularity of solutions in the third section. The proofs are given in the Section 5 after we give two examples which satisfy our assumptions in Section 4, one with space-time white noise and one with trace-class noise.

In conclusion, we note that we assume that the drift coefficient $F$ satisfies a global Lipschitz condition, but this is only a convenience which allows us to focus on the noise terms here. Our results can be extended to more general drift coefficients such as those satisfying a dissipativity condition as in $[1,2,7]$.

\section{Setting and assumptions}

Fix $T>0$ and let $(\Omega, \mathcal{F}, \mathbb{P})$ be a probability space with a normal filtration $\mathcal{F}_{t}, t \in[0, T]$, see for example [3] for details. In addition, let $\left(H,\langle\cdot, \cdot\rangle_{H}\right)$ and $\left(U,\langle\cdot, \cdot\rangle_{U}\right)$ be two separable $\mathbb{R}$-Hilbert spaces with norms denoted by $\|\cdot\|_{H}$ and $\|\cdot\|_{U}$, respectively. Moreover, let $\left(D,\|\cdot\|_{D}\right)$ be a separable $\mathbb{R}$-Banach space with $H \subset D$ continuously and let $L(U, D)$ be the $\mathbb{R}$-Banach space of all bounded linear operators from $U$ to $D$.

We consider the SPDE (1.1) in the mild integral equation form (1.2) on $H$, where $W_{t}, t \in[0, T]$, is a cylindrical $Q$-Wiener process on $U$ with respect to $\mathcal{F}_{t}, t \in[0, T]$ for which the covariance operator $Q=I$ is the identity on $U$ (see [9]) and where the objects $A, F, B$ and $x_{0}$ are specified through the following assumptions. 
ASSUMPTION 2.1 (Linear operator $A$ ). Let $\mathcal{I}$ be a finite or countable set. Moreover, let $\left(\lambda_{i}\right)_{i \in \mathcal{I}}$ be a family of positive real numbers with $\inf _{i \in \mathcal{I}} \lambda_{i}>0$ and let $\left(e_{i}\right)_{i \in \mathcal{I}}$ be an orthonormal basis of $H$. Then, suppose that the linear operator $A: D(A) \subset H \rightarrow H$ is given by

$$
A v=\sum_{i \in \mathcal{I}}-\lambda_{i}\left\langle e_{i}, v\right\rangle_{H} e_{i}
$$

for all $v \in D(A)$ with $D(A)=\left\{v \in H: \sum_{i \in \mathcal{I}}\left|\lambda_{i}\right|^{2}\left|\left\langle e_{i}, v\right\rangle_{H}\right|^{2}<\infty\right\}$.

ASSUMPTION 2.2 (Drift $F$ ). The mapping $F: H \rightarrow H$ is globally Lipschitz continuous with respect to $\|\cdot\|_{H}$.

Let $D\left((-A)^{r}\right), r \in \mathbb{R}$, denote the interpolation spaces of powers of the operator $-A$ (see, for example, [10]) and let $\|\cdot\|_{\text {HS }}$ denote the Hilbert-Schmidt norm for HilbertSchmidt operators from $U$ to $H$.

ASSumption 2.3 (Diffusion $B$ ). Suppose that $D \subset D\left((-A)^{-r}\right)$ continuously for some $r \geq 0$ and that $B: H \rightarrow L(U, D)$ is a strongly measurable mapping such that $e^{A t} B(v)$ is a Hilbert-Schmidt operator from $U$ to $H$ and

$$
\begin{gathered}
\left\|e^{A t} B(v)\right\|_{\mathrm{HS}} \leq L\left(1+\|v\|_{H}\right) t^{(\varepsilon-1 / 2)} \\
\left\|e^{A t}(B(v)-B(w))\right\|_{\mathrm{HS}} \leq L\|v-w\|_{H} t^{(\varepsilon-1 / 2)}
\end{gathered}
$$

for all $v, w \in H$ and $t \in(0, T]$, where $L>0$ and $\varepsilon>0$ are given constants.

By the assumed strong measurability we mean that the mappings

$$
H \rightarrow D, \quad v \mapsto B(v) u, \quad v \in H
$$

are $\mathcal{B}(H) / \mathcal{B}(D)$-measurable for every $u \in U$. If $B: H \rightarrow L(U, D)$ is assumed to be $\mathcal{B}(H) / \mathcal{B}(L(U, D))$-measurable, then in particular it is strongly measurable.

ASSUMPTION 2.4 (Initial value $x_{0}$ ). Let $p \in[2, \infty$ ) be given and suppose that $x_{0}: \Omega \rightarrow H$ is a $\mathcal{F}_{0} / \mathcal{B}(H)$-measurable mapping with $\mathbb{E}\left\|x_{0}\right\|_{H}^{p}<\infty$.

\section{Existence, uniqueness and regularity results}

The main result of this article is that the SPDE (1.1) has a unique solution up to modifications under the assumptions given in Section 2.

THEOREM 3.1. Let assumptions 2.1-2.4 be satisfied. Then, there is a unique (up to modifications) predictable stochastic process $X: \Omega \times[0, T] \rightarrow H$ with $\sup _{0 \leq t \leq T} \mathbb{E}\left\|X_{t}\right\|_{H}^{p}<\infty$, where $p \geq 2$ is given in Assumption 2.4, such that

$$
\mathbb{P}\left[X_{t}=e^{A t} x_{0}+\int_{0}^{t} e^{A(t-s)} F\left(X_{s}\right) d s+\int_{0}^{t} e^{A(t-s)} B\left(X_{s}\right) d W_{s}\right]=1
$$

for all $t \in[0, T]$. Furthermore, $X$ is the unique mild solution of the SPDE (1.1) in this sense. 
Note that the integrals in Equation (3.1) are well defined under the Assumptions 2.1-2.4 (see the proof of Theorem 3.1). Finally, we also obtain the following regularity property of the solution if further assumptions on $e^{A t} B(\cdot)$ are satisfied.

Theorem 3.2. Let Assumptions 2.1-2.4 be satisfied and let $\gamma \in(0,1)$ be such that $\mathbb{E}\left\|(-A)^{\gamma} x_{0}\right\|_{H}^{p}<\infty$. Furthermore, suppose that $(-A)^{\gamma} e^{A t} B(v)$ is a Hilbert-Schmidt operator from $U$ to $H$ with

$$
\left\|(-A)^{\gamma} e^{A t} B(v)\right\|_{\mathrm{HS}} \leq L\left(1+\|v\|_{H}\right) t^{(\varepsilon-1 / 2)}
$$

for all $v \in H$ and all $t \in(0, T]$ with constants $L>0$ and $\varepsilon>0$. Then, the unique solution process $X: \Omega \times[0, T] \rightarrow H$ of the SPDE (1.1) given by Theorem 3.1 satisfies $\sup _{0 \leq t \leq T} \mathbb{E}\left\|(-A)^{\gamma} X_{t}\right\|_{H}^{p}<\infty$.

\section{Examples}

As an example in which our assumptions hold, we consider the following situation. Let $O:=(0,1)^{d} \subset \mathbb{R}^{d}$ with $d \in \mathbb{N}$ and let $H=L^{2}(O, \mathbb{R})$ be the Hilbert space of all square integrable functions from $O$ to $\mathbb{R}$, with scalar product and the norm

$$
\langle v, w\rangle_{H}=\int_{O} v(x) w(x) d x, \quad\|v\|_{H}=\left(\int_{O} v(x)^{2} d x\right)^{1 / 2}
$$

for every $v, w \in H$. (In fact, $H$ is the Hilbert space of equivalence classes of square integrable functions, but for convenience we do not take care of this differentiation.) We also define $U:=H$. Moreover, let $A=\vartheta \Delta$ with $\vartheta>0$ be a constant times the Laplacian with Dirichlet boundary conditions, so $\mathcal{I}=\mathbb{N}^{d}$ and

$$
e_{i}(x)=2^{d / 2} \sin \left(i_{1} \pi x_{1}\right) \cdots \sin \left(i_{d} \pi x_{d}\right), \quad \lambda_{i}=\vartheta \pi^{2}\left(i_{1}^{2}+\cdots+i_{d}^{2}\right)
$$

for all $x=\left(x_{1}, \ldots, x_{d}\right) \in O$ and all $i=\left(i_{1}, \ldots, i_{d}\right) \in \mathcal{I}$. The operator $A$ is then given by

$$
A f=\sum_{i \in \mathcal{I}}-\lambda_{i}\left\langle e_{i}, f\right\rangle_{H} e_{i}
$$

for all $f \in D(A)$ with

$$
D(A)=\left\{\left.f \in H\left|\sum_{i \in \mathcal{I}} \lambda_{i}^{2}\right|\left\langle e_{i}, f\right\rangle_{H}\right|^{2}<\infty\right\} .
$$

Thus, Assumption 2.1 holds here.

Furthermore, let $f, g: \mathbb{R} \rightarrow \mathbb{R}$ be two globally Lipschitz continuous functions, that is,

$$
|g(x)-g(y)| \leq L|x-y|, \quad|f(x)-f(y)| \leq L|x-y|
$$


for all $x, y \in \mathbb{R}$ with a constant $L>0$, and define

$$
F: H \rightarrow H, \quad F(v)(x)=f(v(x)), \quad x \in(0,1)^{d}
$$

and

$$
G: H \rightarrow H, \quad G(v)(x)=g(v(x)), \quad x \in(0,1)^{d}
$$

for all $v \in H$. Hence, $F$ and $G$ are also globally Lipschitz continuous functions on $H$, that is,

$$
\|G(v)-G(w)\|_{H} \leq L\|v-w\|_{H}, \quad\|F(v)-F(w)\|_{H} \leq L\|v-w\|_{H}
$$

for all $v, w \in H$, and Assumption 2.2 holds.

To discuss Assumption 2.3 we now consider separately the two cases of space-time white noise and trace-class noise.

4.1. Space-time white noise. Let $d=1$ and $D=L^{1}(0,1)$. Then, we define $B$ by

$$
B: H \rightarrow L(H, D), \quad(B(v)(w))(x):=(G(v))(x) \cdot w(x)
$$

for every $x \in(0,1)$ and $v, w \in H$. Indeed, $B$ is well defined, since, by the CauchySchwarz inequality,

$$
\begin{aligned}
\|B(v)(w)\|_{D} & =\int_{0}^{1}|G(v)(x) \cdot w(x)| d x \\
& \leq\left(\int_{0}^{1}|G(v)(x)|^{2} d x\right)^{1 / 2}\left(\int_{0}^{1}|w(x)|^{2} d x\right)^{1 / 2}=\|G(v)\|_{H} \cdot\|w\|_{H}
\end{aligned}
$$

for all $v, w \in H$, so $B(v)$ is indeed a bounded linear operator from $H$ to $D$ with the property

$$
\|B(v)\|_{L(H, D)} \leq\|G(v)\|_{H}
$$

for all $v \in H$. In the same way, we obtain

$$
\|B(v)-B(w)\|_{L(H, D)} \leq\|G(v)-G(w)\|_{H} \leq L\|v-w\|_{H}
$$

for all $v, w \in H$, since $G$ is globally Lipschitz continuous. Hence, $B$ is also a globally Lipschitz continuous function from $H$ to $L(H, D)$ and is, in particular, measurable. Combining the definitions in (4.1)-(4.3), we obtain

$$
B: H \rightarrow L(H, D), \quad(B(v)(w))(x):=g(v(x)) \cdot w(x) \quad \forall x \in(0,1)
$$


and every $v, w \in H$. In the next step, let $\gamma \geq 0$. Then, of course, $(-A)^{\gamma} e^{A t} B(v)$ is a bounded linear operator from $H$ to $H$ for every $v \in H$ and $t \in(0, T]$. Moreover,

$$
\begin{aligned}
\left\|(-A)^{\gamma} e^{A t} B(v)\right\|_{\mathrm{HS}}^{2} & =\sum_{i \in \mathcal{I}}\left\|(-A)^{\gamma} e^{A t} B(v) e_{i}\right\|_{H}^{2} \\
& =\sum_{i \in \mathcal{I}}\left\|(-A)^{\gamma} e^{A t}\left(\sum_{j \in \mathcal{I}}\left\langle e_{j}, B(v) e_{i}\right\rangle_{H} e_{j}\right)\right\|_{H}^{2} \\
& =\sum_{i \in \mathcal{I}}\left\|\sum_{j \in \mathcal{I}}(-A)^{\gamma} e^{A t} e_{j}\left\langle e_{j}, B(v) e_{i}\right\rangle_{H}\right\|_{H}^{2} \\
& =\sum_{i \in \mathcal{I}} \sum_{j \in \mathcal{I}}\left(\left(\lambda_{j}\right)^{2 \gamma} e^{-2 \lambda_{j} t}\left|\left\langle e_{j}, B(v) e_{i}\right\rangle_{H}\right|^{2}\right)
\end{aligned}
$$

for all $v \in H, t \in(0, T]$. Therefore, the definition of $B$ yields

$$
\begin{aligned}
\left\|(-A)^{\gamma} e^{A t} B(v)\right\|_{\mathrm{HS}}^{2} & =\sum_{i \in \mathcal{I}} \sum_{j \in \mathcal{I}}\left(\left(\lambda_{j}\right)^{2 \gamma} e^{-2 \lambda_{j} t}\left|\left\langle e_{i}, B(v) e_{j}\right\rangle_{H}\right|^{2}\right) \\
& =\sum_{j \in \mathcal{I}}\left(\left(\lambda_{j}\right)^{2 \gamma} e^{-2 \lambda_{j} t}\left(\sum_{i \in \mathcal{I}}\left|\left\langle e_{i}, B(v) e_{j}\right\rangle_{H}\right|^{2}\right)\right) \\
& =\sum_{j \in \mathcal{I}}\left(\left(\lambda_{j}\right)^{2 \gamma} e^{-2 \lambda_{j} t}\left\|B(v) e_{j}\right\|_{H}^{2}\right)
\end{aligned}
$$

for all $v \in H$ and all $t \in(0, T]$. Since $\left|e_{j}(x)\right| \leq \sqrt{2}$ for every $j \in \mathcal{I}=\mathbb{N}$ and $x \in(0,1)$, we obtain

$$
\begin{aligned}
\left\|(-A)^{\gamma} e^{A t} B(v)\right\|_{\mathrm{HS}} & \leq\left(\sum_{j \in \mathcal{I}}\left(\left(\lambda_{j}\right)^{2 \gamma} e^{-2 \lambda_{j} t} 2\|G(v)\|_{H}^{2}\right)\right)^{1 / 2} \\
& =\sqrt{2}\|G(v)\|_{H}\left\|(-A)^{\gamma} e^{A t}\right\|_{\mathrm{HS}}
\end{aligned}
$$

for all $v \in H, t \in(0, T]$. Suppose now that $\gamma \in\left[0, \frac{1}{4}\right)$. Then, we obtain

$$
\begin{aligned}
\left\|(-A)^{\gamma} e^{A t}\right\|_{\mathrm{HS}}^{2} & =\sum_{j=1}^{\infty}\left(\lambda_{j}\right)^{2 \gamma} \exp \left(-2 \lambda_{j} t\right)=\sum_{j=1}^{\infty}\left(\vartheta j^{2} \pi^{2}\right)^{2 \gamma} \exp \left(-2 \vartheta j^{2} \pi^{2} t\right) \\
& \leq \vartheta^{2 \gamma} \pi^{4 \gamma}\left(\int_{0}^{\infty}\left(x^{4 \gamma}+1\right) \exp \left(-\frac{1}{2}(2 \sqrt{\vartheta t} \pi x)^{2}\right) d x\right) \\
& \leq \frac{\vartheta^{2 \gamma} \pi^{4 \gamma}}{2 \sqrt{\vartheta t} \pi}\left(\int_{-\infty}^{\infty}\left(\left(\frac{|x|}{2 \sqrt{\vartheta t} \pi}\right)^{4 \gamma}+1\right) \exp \left(-x^{2} / 2\right) d x\right) \\
& =\frac{\vartheta^{\left(2 \gamma-\frac{1}{2}\right)} \pi^{(4 \gamma-1)}}{2 \sqrt{t}}\left(\int_{-\infty}^{\infty}\left(\frac{|x|}{2 \sqrt{\vartheta t} \pi}\right)^{4 \gamma} \exp \left(-x^{2} / 2\right) d x+\sqrt{2 \pi}\right)
\end{aligned}
$$


for every $t \in(0, T]$. Hence,

$$
\begin{aligned}
\left\|(-A)^{\gamma} e^{A t}\right\|_{\mathrm{HS}}^{2} & \leq \frac{\vartheta^{\left(2 \gamma-\frac{1}{2}\right)} \sqrt{\pi}}{\sqrt{2 t}}\left(\frac{1}{\sqrt{2 \pi}} \int_{-\infty}^{\infty}\left(\frac{|x|}{\sqrt{\vartheta t}}\right)^{4 \gamma} e^{-x^{2} / 2} d x+1\right) \\
& =\frac{\vartheta^{\left(2 \gamma-\frac{1}{2}\right)} \sqrt{\pi}}{\sqrt{2 t}}\left(\frac{(\vartheta t)^{-2 \gamma}}{\sqrt{2 \pi}} \int_{-\infty}^{\infty}|x|^{4 \gamma} e^{-x^{2} / 2} d x+1\right) \\
& \leq \frac{\vartheta^{\left(2 \gamma-\frac{1}{2}\right)} \sqrt{\pi}}{\sqrt{2 t}}\left(\frac{(\vartheta t)^{-2 \gamma}}{\sqrt{2 \pi}} \int_{-\infty}^{\infty}\left(1+x^{2}\right) e^{-x^{2} / 2} d x+1\right) \\
& =\frac{\vartheta^{\left(2 \gamma-\frac{1}{2}\right)} \sqrt{\pi}}{\sqrt{2 t}}\left(2(\vartheta t)^{-2 \gamma}+1\right) \leq \frac{\vartheta^{\left(2 \gamma-\frac{1}{2}\right)} \sqrt{2 \pi}}{\sqrt{t}}\left((\vartheta t)^{-2 \gamma}+1\right) \\
& =\sqrt{2 \pi}\left(\vartheta^{-\frac{1}{2}} t^{\left(-\frac{1}{2}-2 \gamma\right)}+\vartheta^{\left(-\frac{1}{2}+2 \gamma\right)} t^{-\frac{1}{2}}\right) \\
& \leq \frac{\sqrt{2 \pi}\left(1+T^{2 \gamma}\right)}{\min (\vartheta, 1)} t^{\left(-\frac{1}{2}-2 \gamma\right)}
\end{aligned}
$$

which yields

$$
\left\|(-A)^{\gamma} e^{A t}\right\|_{\mathrm{HS}} \leq\left(\frac{\sqrt{\sqrt{2 \pi}} \sqrt{T+2}}{\min (\vartheta, 1)}\right) t^{-\left(\frac{1}{4}+\gamma\right)}
$$

for every $t \in(0, T]$. Hence, we obtain

$$
\left\|(-A)^{\gamma} e^{A t} B(v)\right\|_{\mathrm{HS}} \leq\left(\frac{4(T+1)}{\min (\vartheta, 1)}\right)\|G(v)\|_{H} t^{-\left(\frac{1}{4}+\gamma\right)}
$$

for every $v \in H, t \in(0, T]$ and $\gamma \in\left[0, \frac{1}{4}\right)$. In the same way, we can show that

$$
\left\|(-A)^{\gamma} e^{A t}(B(v)-B(w))\right\|_{\mathrm{HS}} \leq\left(\frac{4(T+1)}{\min (\vartheta, 1)}\right)\|G(v)-G(w)\|_{H} t^{-\left(\frac{1}{4}+\gamma\right)}
$$

for every $v, w \in H, t \in(0, T]$ and $\gamma \in\left[0, \frac{1}{4}\right)$. Thus, Assumption 2.3 holds. Finally, if the initial value satisfies Assumption 2.4, then the SPDE

$$
d X_{t}(x)=\left[\Delta X_{t}(x)+f\left(X_{t}(x)\right)\right] d t+g\left(X_{t}(x)\right) d W_{t}, \quad X_{0}(x)=x_{0}(x), x \in(0,1)
$$

has a unique solution by Theorem 3.1 and if the initial value also satisfies

$$
\mathbb{E}\left\|(-A)^{\gamma} x_{0}\right\|^{p}<\infty \quad \text { with } \gamma \in\left[0, \frac{1}{4}\right) \text { and } p \in[2, \infty),
$$

then the solution has values in $D\left((-A)^{\gamma}\right)$ almost surely in the sense of Theorem 3.2. In particular, the equation with additive noise

$$
d X_{t}(x)=\left[\Delta X_{t}(x)+f\left(X_{t}(x)\right)\right] d t+d W_{t}, \quad x \in(0,1)
$$

with $g(y) \equiv 1$ and the stochastic heat equation with linear multiplicative noise

$$
d X_{t}(x)=\Delta X_{t}(x) d t+X_{t}(x) d W_{t}, \quad x \in(0,1)
$$

with $g(y)=y$ and $f(y)=0$ for all $y \in \mathbb{R}$ both have unique solutions. 
4.2. Trace-class noise. Let $d \in \mathbb{N}, D=H$ and let $\left(f_{i}\right)_{i \in \mathcal{I}}$ be another orthonormal basis in $H$ with the property that $f_{i}: \bar{O} \rightarrow \mathbb{R}$ are continuous functions, which satisfy

$$
\sup _{i \in \mathcal{I}} \sup _{x \in \bar{O}}\left|f_{i}(x)\right|<\infty
$$

Moreover, let $\sqrt{Q}: H \rightarrow H$ be a bounded linear operator given by

$$
\sqrt{Q} v=\sum_{i \in \mathcal{I}} \alpha_{i} f_{i}\left\langle f_{i}, v\right\rangle_{H}, \quad v \in H
$$

for real numbers $\left(\alpha_{i}\right)_{i \in \mathcal{I}}$ satisfying $\sum_{i \in \mathcal{I}} \alpha_{i}^{2}<\infty$. Then $\sqrt{Q} v: \bar{O} \rightarrow H$ is a continuous mapping with

$$
\sup _{x \in \bar{O}}|(\sqrt{Q} v)(x)| \leq c\|v\|_{H}=c \sqrt{\int_{O}|v(x)|^{2} d x}
$$

for every $v \in H$, where the constant $c \in[0, \infty)$ is given by

$$
c=\left(\left(\sum_{i \in I} \alpha_{i}^{2}\right)^{1 / 2}\left(\sup _{i \in \mathcal{I}} \sup _{x \in \bar{O}}\left|f_{i}(x)\right|\right)\right)=\|\sqrt{Q}\|_{\mathrm{HS}}\left(\sup _{i \in \mathcal{I}} \sup _{x \in \bar{O}}\left|f_{i}(x)\right|\right) .
$$

Indeed, for $v \in H$, we obtain

$$
\begin{aligned}
\sup _{x \in \bar{O}}|(\sqrt{Q} v)(x)| & =\sup _{x \in \bar{O}}\left|\sum_{i \in I} \alpha_{i} f_{i}(x)\left\langle f_{i}, v\right\rangle_{H}\right| \\
& \leq \sum_{i \in I}\left(\left|\alpha_{i}\right| \cdot\left|\left\langle f_{i}, v\right\rangle_{H}\right| \cdot \sup _{x \in \bar{O}}\left|f_{i}(x)\right|\right) \\
& \leq \sum_{i \in I}\left(\left|\alpha_{i}\right| \cdot\left|\left\langle f_{i}, v\right\rangle_{H}\right|\right)\left(\sup _{i \in \mathcal{I}} \sup _{x \in \bar{O}}\left|f_{i}(x)\right|\right) \\
& \leq\left(\sum_{i \in I} \alpha_{i}^{2}\right)^{1 / 2}\left(\sum_{i \in I}\left|\left\langle f_{i}, v\right\rangle_{H}\right|^{2}\right)^{1 / 2}\left(\sup _{i \in \mathcal{I}} \sup _{x \in \bar{O}}\left|f_{i}(x)\right|\right) \\
& \leq\left(\sum_{i \in I} \alpha_{i}^{2}\right)^{1 / 2}\left(\sup _{i \in \mathcal{I}} \sup _{x \in \bar{O}}\left|f_{i}(x)\right|\right)\|v\|_{H}=c\|v\|_{H} .
\end{aligned}
$$

In the next step, we define $B$ by

$$
B: H \rightarrow L(H, D), \quad(B(v)(w))(x):=(G(v))(x) \cdot(\sqrt{Q} w)(x)
$$


for every $x \in(0,1)^{d}$ and $v, w \in H$. Indeed, $B$ is well defined, since

$$
\begin{aligned}
\|B(v)(w)\|_{D} & =\left(\int_{(0,1)^{d}}|G(v)(x) \cdot(\sqrt{Q} w)(x)|^{2} d x\right)^{1 / 2} \\
& \leq\left(\int_{(0,1)^{d}}|G(v)(x)|^{2} d x\right)^{1 / 2}\left(\sup _{x \in \bar{O}}|(\sqrt{Q} w)(x)|\right) \\
& =\|G(v)\|_{H}\left(\sup _{x \in \bar{O}}|(\sqrt{Q} w)(x)|\right) \leq c\|G(v)\|_{H}\|w\|_{H}
\end{aligned}
$$

for all $v, w \in H$. Therefore, $B(v)$ is a bounded linear operator from $H$ to $H=D$ with the property

$$
\|B(v)\|_{L(H, D)} \leq c\|G(v)\|_{H}
$$

for all $v \in H$. In the same way, we obtain

$$
\|B(v)-B(w)\|_{L(H, D)} \leq c\|G(v)-G(w)\|_{H} \leq c L\|v-w\|_{H}
$$

for all $v, w \in H$, since $G$ is globally Lipschitz continuous. Hence, $B$ is also a globally Lipschitz continuous function from $H$ to $L(H, H)$. Combining the definitions in (4.1)-(4.2) and (4.4), we obtain that the operator $B: H \rightarrow L(H, D)$ is defined by

$$
(B(v)(w))(x):=g(v(x)) \cdot(\sqrt{Q} w)(x) \quad x \in(0,1)^{d}
$$

for every $v, w \in H$. Let $\gamma \in[0,1)$. Hence, $(-A)^{\gamma} e^{A t} B(v)$ is a linear bounded operator from $H$ to $H$ and

$$
\begin{aligned}
\left\|(-A)^{\gamma} e^{A t} B(v)\right\|_{\mathrm{HS}} & \leq\left\|(-A)^{\gamma} e^{A t}\right\|_{L(H, H)}\|B(v)\|_{\mathrm{HS}} \leq t^{-\gamma}\|B(v)\|_{\mathrm{HS}} \\
& =t^{-\gamma}\left(\sum_{i \in \mathcal{I}}\left\|B(v)\left(f_{i}\right)\right\|_{H}^{2}\right)^{1 / 2} \\
& =t^{-\gamma}\left(\sum_{i \in \mathcal{I}} \alpha_{i}^{2}\left\|B(v)\left(\frac{f_{i}}{\alpha_{i}}\right)\right\|_{H}^{2}\right)^{1 / 2} \\
& \leq t^{-\gamma}\left(\sum_{i \in \mathcal{I}} \alpha_{i}^{2}\right)^{1 / 2}\left(\sup _{i \in \mathcal{I}}\left\|B(v)\left(\frac{f_{i}}{\alpha_{i}}\right)\right\|_{H}\right) \\
& \leq t^{-\gamma}\left(\sum_{i \in \mathcal{I}} \alpha_{i}^{2}\right)^{1 / 2}\left(\sup _{i \in \mathcal{I}}\|G(v)\|_{H}\left(\sup _{x \in \bar{O}}\left|\left(\sqrt{Q} \frac{f_{i}}{\alpha_{i}}\right)(x)\right|\right)\right) \\
& =t^{-\gamma}\left(\sum_{i \in \mathcal{I}} \alpha_{i}^{2}\right)^{1 / 2}\|G(v)\|_{H}\left(\sup _{i \in \mathcal{I}} \sup _{x \in \bar{O}}\left|f_{i}(x)\right|\right) \\
& =c\|G(v)\|_{H} t^{-\gamma}
\end{aligned}
$$

for every $t>0$ and $v \in H$ (see also [8, Remark 1]). In the same way, we obtain

$$
\left\|(-A)^{\gamma} e^{A t}(B(v)-B(w))\right\|_{\mathrm{HS}} \leq c\|G(v)-G(w)\| t^{-\gamma}
$$


for every $t>0$ and every $v, w \in H$. This yields that Assumption 2.3 is fulfilled. Finally, if the initial value satisfies Assumption 2.4, then the SPDE

$$
d X_{t}(x)=\left[\Delta X_{t}(x)+f\left(X_{t}(x)\right)\right] d t+g\left(X_{t}(x)\right) \sqrt{Q} d W_{t}, \quad X_{0}(x)=x_{0}(x)
$$

with $x \in(0,1)^{d}$ has a unique solution by Theorem 3.1 and if the initial value also satisfies $\mathbb{E}\left\|(-A)^{\gamma} x_{0}\right\|_{H}^{p}<\infty$ with $\gamma \in\left[0, \frac{1}{2}\right)$, then the solution has values in $D\left((-A)^{\gamma}\right)$ almost surely in the sense of Theorem 3.2. In particular, the equation with additive noise

$$
d X_{t}(x)=\left[\Delta X_{t}(x)+f\left(X_{t}(x)\right)\right] d t+\sqrt{Q} d W_{t}, \quad x \in(0,1)^{d}
$$

with $g(y) \equiv 1$ and the stochastic heat equation with linear multiplicative noise

$$
d X_{t}(x)=\Delta X_{t}(x) d t+X_{t}(x) \sqrt{Q} d W_{t}, \quad x \in(0,1)^{d}
$$

with $g(y)=y$ and $f(y)=0$ for all $y \in \mathbb{R}$ both have unique solutions.

\section{Proofs}

Let $(V,\|\cdot\|)$ be a normed vector space and denote the $L^{q}$-norm for $q \in[1, \infty)$ of a $\mathcal{F} / \mathcal{B}(V)$-measurable mapping $Z: \Omega \rightarrow V$ by $\|Z\|_{L^{q}}:=\left(\mathbb{E}\|Z\|^{q}\right)^{1 / q}$. We need the following version of the Burkholder-Davis-Gundy inequality in infinite dimensions (see [3, Lemma 7.7]).

LEMMA 5.1. Let $X:[0, T] \times \Omega \rightarrow H S(U, H)$ be a predictable stochastic process with $\mathbb{E} \int_{0}^{T}\left\|X_{s}\right\|_{\mathrm{HS}}^{2} d s<\infty$. Then, we obtain

$$
\left\|\int_{0}^{t} X_{s} d W_{s}\right\|_{L^{q}} \leq q\left(\int_{0}^{t}\|\| X_{s}\left\|_{H S}\right\|_{L^{q}}^{2} d s\right)^{1 / 2}
$$

for every $t \in[0, T]$ and every $q \in[2, \infty)$. Both sides could be infinite.

Proof of TheOREM 3.1. Let $p \geq 2$ be given by Assumption 2.4. First, we introduce the $\mathbb{R}$-vector space $\mathcal{V}_{p}$ of all equivalence classes of predictable stochastic processes $X: \Omega \times[0, T] \rightarrow H$ with $\sup _{0 \leq t \leq T}\left\|X_{t}\right\|_{L^{p}}<\infty$, where all stochastic processes that are modifications of each other lie in one equivalence class. Then, we equip this space with the norms $\|X\|_{\mu}:=\sup _{0 \leq t \leq T} e^{\mu t}\left\|X_{t}\right\|_{L^{p}}$ for every $X \in \mathcal{V}_{p}$ and every $\mu \in \mathbb{R}$. Note that the pair $\left(\mathcal{V}_{p},\|\cdot\|_{\mu}\right)$ is a Banach space for every $\mu \in \mathbb{R}$.

Now, we consider the mapping $\phi: \mathcal{V}_{p} \rightarrow \mathcal{V}_{p}$ given by

$$
(\phi X)_{t}:=e^{A t} x_{0}+\int_{0}^{t} e^{A(t-s)} F\left(X_{s}\right) d s+\int_{0}^{t} e^{A(t-s)} B\left(X_{s}\right) d W_{s}
$$

for every $t \in[0, T]$ and $X \in \mathcal{V}_{p}$. First, we show that $\phi$ is well defined. Given $t \in[0, T]$ and $X \in \mathcal{V}_{p}$, the mapping from $[0, t] \times \Omega$ to $\operatorname{HS}(U, H)$ (the space of Hilbert-Schmidt operators from $U$ to $H$ ) defined by

$$
(s, \omega) \rightarrow e^{A(t-s)} B\left(X_{s}(\omega)\right)
$$


for every $s \in[0, t]$ and $\omega \in \Omega$ is a predictable stochastic process, since $e^{A s}$ is continuous in $L(D, H)$ for $s \in(0, T]$ and since $B(\cdot)$ is strongly measurable by Assumption 2.3. Hence,

$$
\begin{aligned}
\left\|\int_{0}^{t} e^{A(t-s)} B\left(X_{S}\right) d W_{s}\right\|_{L^{p}} & \leq p\left(\int_{0}^{t}\|\| e^{A(t-s)} B\left(X_{S}\right)\left\|_{\mathrm{HS}}\right\|_{L^{p}}^{2} d s\right)^{1 / 2} \\
& \leq p\left(\int_{0}^{t}\left\|L\left(1+\left\|X_{s}\right\|_{H}\right)(t-s)^{(\varepsilon-1 / 2)}\right\|_{L^{p}}^{2} d s\right)^{1 / 2} \\
& \leq L p\left(\int_{0}^{t}\left(1+\left\|X_{s}\right\|_{L^{p}}\right)^{2}(t-s)^{(2 \varepsilon-1)} d s\right)^{1 / 2}
\end{aligned}
$$

due to Lemma 5.1 and Assumption 2.3 and, therefore, we obtain

$$
\begin{aligned}
\left\|\int_{0}^{t} e^{A(t-s)} B\left(X_{s}\right) d W_{s}\right\|_{L^{p}} & \leq L p\left(1+\sup _{0 \leq s \leq T}\left\|X_{s}\right\|_{L^{p}}\right)\left(\int_{0}^{t} s^{(2 \varepsilon-1)} d s\right)^{1 / 2} \\
& \leq \frac{L p}{\sqrt{2 \varepsilon}}\left(1+\sup _{0 \leq s \leq T}\left\|X_{s}\right\|_{L^{p}}\right) t^{\varepsilon}<\infty
\end{aligned}
$$

for every $t \in(0, T]$. By Lebesgue's theorem one can show that $\int_{0}^{t} e^{A(t-s)} B\left(X_{S}\right) d W_{S}$ for $t \in[0, T]$ is mean square continuous and, therefore, it has a predictable version. The calculation for the second integral in the definition of $\phi$ is similar and, hence, we obtain that $\phi$ is well defined.

Now, we show that $\phi$ is a contraction with respect to $\|\cdot\|_{\mu}$ for an appropriate $\mu \in \mathbb{R}$. From Assumption 2.2,

$$
\|F(v)-F(w)\|_{H} \leq K\|v-w\|_{H}
$$

for all $v, w \in H$ with a constant $K>0$. Hence, for $X, Y \in \mathcal{V}_{p}$ and $t \in[0, T]$ we obtain

$$
\begin{aligned}
(\phi X)_{t}-(\phi Y)_{t}=\int_{0}^{t} e^{A(t-s)}\left(F\left(X_{s}\right)-F\left(Y_{s}\right)\right) d s & \\
& +\int_{0}^{t} e^{A(t-s)}\left(B\left(X_{s}\right)-B\left(Y_{s}\right)\right) d W_{s} \quad \text { almost surely, }
\end{aligned}
$$

from which it follows that

$$
\begin{aligned}
\left\|(\phi X)_{t}-(\phi Y)_{t}\right\|_{L^{p}} \leq & \int_{0}^{t}\left\|e^{A(t-s)}\left(F\left(X_{s}\right)-F\left(Y_{s}\right)\right)\right\|_{L^{p}} d s \\
& \quad+\left\|\int_{0}^{t} e^{A(t-s)}\left(B\left(X_{s}\right)-B\left(Y_{s}\right)\right) d W_{s}\right\|_{L^{p}} \\
& \leq K\left(\sup _{0 \leq s \leq T}\left\|e^{A s}\right\|_{L(H, H)}\right) \int_{0}^{t}\left\|X_{S}-Y_{S}\right\|_{L^{p}} d s
\end{aligned}
$$




$$
\begin{gathered}
+p\left(\int_{0}^{t}\|\| e^{A(t-s)}\left(B\left(X_{s}\right)-B\left(Y_{s}\right)\right)\left\|_{\mathrm{HS}}\right\|_{L^{p}}^{2} d s\right)^{1 / 2} \\
\leq K\left(\int_{0}^{t} e^{-\mu s} d s\right)\|X-Y\|_{\mu} \\
+p\left(\int_{0}^{t}\left\|L(t-s)^{(\varepsilon-1 / 2)}\right\| X_{s}-Y_{s}\left\|_{H}\right\|_{L^{p}}^{2} d s\right)^{1 / 2}
\end{gathered}
$$

for $\mu \in \mathbb{R}$ by Lemma 5.1. This yields

$$
\begin{aligned}
& e^{\mu t}\left\|(\phi X)_{t}-(\phi Y)_{t}\right\|_{L^{p}} \leq K\left(\int_{0}^{t} e^{\mu s} d s\right)\|X-Y\|_{\mu} \\
& \quad+L p\left(\int_{0}^{t}(t-s)^{(2 \varepsilon-1)} e^{2 \mu t}\left\|X_{s}-Y_{s}\right\|_{L^{p}}^{2} d s\right)^{1 / 2} \\
& \leq \frac{K}{\mu}\left(e^{\mu t}-1\right)\|X-Y\|_{\mu} \\
& \quad+L p\left(\int_{0}^{t}(t-s)^{(2 \varepsilon-1)} e^{2 \mu(t-s)} d s\right)^{1 / 2}\|X-Y\|_{\mu}
\end{aligned}
$$

for every $\mu<0, t \in(0, T]$ and, hence,

$$
\begin{aligned}
\|\phi X-\phi Y\|_{\mu} & \leq \frac{K}{|\mu|}\|X-Y\|_{\mu}+L p\left(\int_{0}^{T} s^{(2 \varepsilon-1)} e^{2 \mu s} d s\right)^{1 / 2}\|X-Y\|_{\mu} \\
& \leq\left(\frac{K}{|\mu|}+L p \sqrt{\int_{0}^{T} s^{(2 \varepsilon-1)} e^{2 \mu s} d s}\right)\|X-Y\|_{\mu}
\end{aligned}
$$

for every $\mu<0$.

Finally, for $\mu \rightarrow-\infty$, we see that $\phi$ is a contraction with respect to $\|\cdot\|_{\mu}$, so there is a unique element $X \in \mathcal{V}_{p}$ with $X=\phi X$. This is the assertion that was to be proved.

Proof of TheOREM 3.2. The solution process $X$ satisfies

$$
\mathbb{P}\left[X_{t}=e^{A t} x_{0}+\int_{0}^{t} e^{A(t-s)} F\left(X_{s}\right) d s+\int_{0}^{t} e^{A(t-s)} B\left(X_{s}\right) d W_{s}\right]=1
$$

for every $t \in[0, T]$.

First,

$$
\left\|(-A)^{\gamma} e^{A t} x_{0}\right\|_{L^{p}}=\left\|e^{A t}(-A)^{\gamma} x_{0}\right\|_{L^{p}} \leq\left\|(-A)^{\gamma} x_{0}\right\|_{L^{p}}<\infty
$$

for every $t \in[0, T]$, where $p \geq 2$ is given in Assumption 2.4. 
Next, we obtain

$$
\begin{aligned}
\left\|(-A)^{\gamma} \int_{0}^{t} e^{A(t-s)} F\left(X_{s}\right) d s\right\|_{L^{p}} & \leq \int_{0}^{t}\left\|(-A)^{\gamma} e^{A(t-s)} F\left(X_{s}\right)\right\|_{L^{p}} d s \\
& \leq \int_{0}^{t}\left\|(-A)^{\gamma} e^{A(t-s)}\right\|_{L(H, H)}\left\|F\left(X_{S}\right)\right\|_{L^{p}} d s \\
& \leq \int_{0}^{t}(t-s)^{-\gamma} K\left(1+\left\|X_{s}\right\|_{L^{p}}\right) d s
\end{aligned}
$$

for $t \in[0, T]$, where we used the fact that $\|F(v)\|_{H} \leq K\left(1+\|v\|_{H}\right)$ for all $v \in H$ with a constant $K>0$. This yields

$$
\begin{aligned}
\left\|(-A)^{\gamma} \int_{0}^{t} e^{A(t-s)} F\left(X_{s}\right) d s\right\|_{L^{p}} & \leq K\left(1+\sup _{0 \leq s \leq T}\left\|X_{S}\right\|_{L^{p}}\right)\left(\int_{0}^{t} s^{-\gamma} d s\right) \\
& \leq K\left(1+\sup _{0 \leq s \leq T}\left\|X_{S}\right\|_{L^{p}}\right) \frac{t^{(1-\gamma)}}{(1-\gamma)} \\
& \leq \frac{K(T+1)}{(1-\gamma)}\left(1+\sup _{0 \leq s \leq T}\left\|X_{S}\right\|_{L^{p}}\right)<\infty
\end{aligned}
$$

for every $t \in[0, T]$. Finally, we obtain

$$
\begin{aligned}
\left\|(-A)^{\gamma} \int_{0}^{t} e^{A(t-s)} B\left(X_{S}\right) d W_{S}\right\|_{L^{p}} & \leq p\left(\int_{0}^{t}\|\|(-A)^{\gamma} e^{A(t-s)} B\left(X_{s}\right)\left\|_{\mathrm{HS}}\right\|_{L^{p}}^{2} d s\right)^{1 / 2} \\
& \leq p\left(\int_{0}^{t}\left\|L\left(1+\left\|X_{s}\right\|_{H}\right)(t-s)^{(\varepsilon-1 / 2)}\right\|_{L^{p}}^{2} d s\right)^{1 / 2} \\
& \leq L p\left(1+\sup _{0 \leq s \leq T}\left\|X_{s}\right\|_{L^{p}}\right)\left(\int_{0}^{t} s^{(2 \varepsilon-1)} d s\right)^{1 / 2} \\
& \leq L p\left(1+\sup _{0 \leq s \leq T}\left\|X_{S}\right\|_{L^{p}}\right) \frac{T^{\varepsilon}}{\sqrt{2 \varepsilon}}<\infty
\end{aligned}
$$

for all $t \in[0, T]$ due to [3, Proposition 4.15] and Lemma 5.1, which yields the assertion.

\section{References}

[1] A. Chojnowska-Michalik and B. Goldys, 'Existence, uniqueness and invariant measures for stochastic semilinear equations on Hilbert spaces', Probab. Theory Related Fields 102 (1995), 331-356.

[2] G. Da Prato, A. Debussche and B. Goldys, 'Some properties of invariant measures of non symmetric dissipative stochastic systems', Probab. Theory Related Fields 123 (2002), 355-380.

[3] G. Da Prato and J. Zabczyk, Stochastic Equations in Infinite Dimensions (Cambridge University Press, Cambridge, 1992). 
[4] G. Da Prato and J. Zabczyk, Ergodicity for Infinite Dimensional Systems, London Mathematical Society Lecture Notes Series, 229 (Cambridge University Press, Cambridge, 1996).

[5] A. Jentzen, 'Taylor expansions of solutions of stochastic partial differential equations', Preprint, 2009.

[6] A. Jentzen and P. E. Kloeden, 'The numerical approximation of stochastic partial differential equations', Milan J. Math., (2009) to appear.

[7] R. Manthey and T. Zausinger, 'Stochastic evolution equations in $L_{\rho}^{2 v}$ ', Stoch. Stoch. Rep. 66 (1999), 37-85.

[8] T. Müller-Gronbach and K. Ritter, 'Lower bounds and nonuniform time discretization for approximation of stochastic heat equations', Found. Comput. Math. 7 (2007), 135-181.

[9] C. Prévot and M. Röckner, A Concise Course on Stochastic Partial Differential Equations (Springer, Berlin, 2007).

[10] G. R. Sell and Y. You, Dynamics of Evolutionary Equations (Springer, New York, 2002).
A. JENTZEN, Institut für Mathematik, Johann Wolfgang Goethe-Universität,
D-60054 Frankfurt am Main, Germany
e-mail: jentzen@math.uni-frankfurt.de
P. E. KLOEDEN, Institut für Mathematik, Johann Wolfgang Goethe-Universität,
D-60054 Frankfurt am Main, Germany
e-mail: kloeden@math.uni-frankfurt.de 